\title{
Partition Testing in Dose-Response Studies with Multiple Endpoints
}

\author{
Yi Liu ${ }^{1}$, Jason $\mathrm{Hsu}^{1}$, and Stephen Ruberg ${ }^{2}$ \\ ${ }^{1}$ Statistics, The Ohio State University, Columbus, OH, USA \\ ${ }^{2}$ Eli Lilly and Company, Indianapolis, Indiana, USA
}

September 21, 2007

\begin{abstract}
Dose-response studies with multiple endpoints can be formulated as closed testing or partition testing problems. When the endpoints are primary and secondary, whether the order in which the doses are to be tested is pre-determined or sample-determined lead to different partitioning of the parameter space corresponding to the null hypotheses to be tested. We use the case of two doses and two endpoints to illustrate how to apply the partitioning principle to construct multiple tests that control the appropriate error rate. Graphical representation can be useful in visualizing the decision process.
\end{abstract}

\section{Historical aspect of dose-response trials in pharmaceutics}

Pharmaceutical drug development has long been divided into four Phases with Phase II being the part of the development cycle where dose response studies are conducted. The goal is usually to 
identify the best dose to use in confirmatory Phase III trials. While this has been the framework for several decades, there are still many drugs that fail to confirm efficacy and safety in Phase III (estimates range from 30-50\%) with a substantial number of failures attributed to improper dose selection. Thus, the importance of the design and analysis of dose response studies is as relevant today as it has ever been.

In the past, efficacy of a new drug was typically demonstrated by showing its superiority to a placebo (called a negative control). However, in recent years, when treatments known to be effective exist, and the disease does not cause mortality or irreversible morbidity, efficacy of a new drug might be defined as superiority or non-inferiority to a known effective treatment (called an active control). In the case of non-inferiority trials, the determination of non-inferiority might be based on what is a clinically meaningful difference. See [1]. Even in the case of superiority trials against a negative control, the definition of superiority might consider risk versus benefit, if there is toxicity concerns for the drug. (see [2].) Some early discussion of these concepts can be seen in $[3,4,5,6]$.

Dose-response studies may have multiple endpoints. A primary endpoint is one such that efficacy of a new drug relative to the control in this single endpoint constitutes evidence of efficacy. Secondary endpoints are ones that efficacy of a new treatment in any secondary endpoints supports evidence of efficacy, but by themselves (i.e. in the absence of efficacy in a primary endpoint) do not constitute evidence of efficacy.

When there are primary and secondary endpoints, inference on the secondary endpoint is given only if the compound is efficacious for the primary endpoint at that dose. This ordering guides the selection of test statistics for each intersection hypothesis in closed testing (as in [7]). We give a different perspective in this article, which is the ordering guides the partitioning of the parameter 
space in using the partitioning principle to construct multiple tests that control the appropriate FWER.

In some (but not all) dose-response studies, it may be appropriate to pre-determine the order in which inferences on the doses are given. For example, one might start with the high dose and proceed to inference on the low dose only if the high dose shows efficacy. We show how this second “ordering" further guides the partitioning of the parameter space. The resulting partitioning test is in the form of a decision tree which can be represented graphically.

Interestingly, the joint distribution of $\mathrm{t}$ test statistics for multiple endpoints is not what is usually called the multivariate $t$ distribution. This article discusses the computation of this distribution in the bivariate case which, to avoid confusion, we call the dual t distribution. We show, for example, that using algorithms for multivariate $t$ distributions results in slightly liberal critical values, while computing as if the $t$ statistics were independent results in somewhat conservative critical values.

Section 2 gives a motivating example of a dose-response study with multiple endpoints. Section 3 shows how the partitioning principle forms null hypotheses when inferences are ordered by dose, and the corresponding multiple test is a step-down test. Section 4 extends the partitioning principle of forming null hypotheses to when inferences are ordered by dose and by endpoint. The corresponding multiple test has a graphical representation. That section also contains a study of issues in the computation of critical values. Section 5 provides a numerical illustration of methods developed in this article, using the real data example in section 2. 


\section{A motivating example for dose-response studies}

Consider, for example, [8], a 26-center double-blind trial comparing the effect of five doses of the anti-psychotic drug "Seroquel" (Quetiapine) and the placebo with parallel design on a total of 361 patients. For illustration purpose, we will focus on two of the doses $(75 \mathrm{mg} /$ day and $600 \mathrm{mg} / \mathrm{day})$ with the primary endpoint being Clinical Global Impression (CGI) Global Improvement score and the secondary endpoint being CGI Severity of Illness score. Summary statistics for these two doses and endpoints are presented in Table 1.

Table 1: Summary statistics of two doses and two endpoints in [8]

\begin{tabular}{|ccccc|}
\hline & & \multicolumn{3}{c|}{ Dose } \\
\cline { 3 - 5 } Endpoint & & $0 \mathrm{mg} /$ day (Placebo) & $75 \mathrm{mg} / \mathrm{day}$ & $600 \mathrm{mg} /$ day \\
\hline & Sample size & 51 & 52 & 51 \\
CGI Global Improvement score & Mean & 4.78 & 4.22 & 3.58 \\
(Primary) & SE $^{\mathrm{a}}$ & 0.23 & 0.22 & 0.23 \\
\hline CGI Severity of Illness score & Mean & 5.2 & 4.8 & 4.4 \\
(Secondary) & SE & 1.2 & 1.3 & 1.5 \\
\hline
\end{tabular}

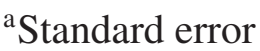

Throughout the paper, the placebo $(0 \mathrm{mg} /$ day $)$, low dose $(75 \mathrm{mg} /$ day $)$ and high dose $(600$ $\mathrm{mg} /$ day) groups will be indexed as $i=0,1,2$. Primary and secondary endpoints will be indexed by superscripts $L=P, S$. For discussion involving only the primary endpoint, the superscript $P$ will be dropped for convenience.

Let $\mu_{i}^{L}$ denote the mean response of dose group $i$ for endpoint $L, i=0,1,2, L=P, S$. Define 
$\theta_{i}^{L}=\mu_{i}^{L}-\mu_{0}^{L}$ as the true mean difference between dose group $i$ and the placebo for endpoint $L$, $i=0,1,2, L=P, S$. Let $\delta^{L}$ denote the clinically meaningful difference for endpoint $L, L=P, S$. The family of null hypotheses of interest consists of four null hypotheses with the first two in (1) concerning primary endpoint and the second two in (2) concerning secondary endpoint.

$$
\begin{array}{lll}
H_{01}^{P}: \theta_{1}^{P} \leq \delta^{P} \quad \text { vs. } \quad H_{a 1}^{P}: \theta_{1}^{P}>\delta^{P} \\
H_{02}^{P}: \theta_{2}^{P} \leq \delta^{P} \quad \text { vs. } \quad H_{a 2}^{P}: \theta_{2}^{P}>\delta^{P} \\
H_{01}^{S}: \theta_{1}^{S} \leq \delta^{S} \quad \text { vs. } \quad H_{a 1}^{S}: \theta_{1}^{S}>\delta^{S} \\
H_{02}^{S}: \theta_{2}^{S} \leq \delta^{S} \quad \text { vs. } \quad H_{a 2}^{S}: \theta_{2}^{S}>\delta^{S}
\end{array}
$$

Assume the samples from dose group $i$ for endpoint $L, Y_{i 1}^{L}, \ldots, Y_{i n_{i}}^{L}, i=0,1,2, L=P, S$, come from a model:

$$
Y_{i r}^{L}=\mu_{i}^{L}+\epsilon_{i r}^{L}, i=0,1,2, r=1, \ldots, n_{i}, L=P, S,
$$

where $\left(\begin{array}{c}\epsilon_{i r}^{P} \\ \epsilon_{i r}^{S}\end{array}\right) \quad$ i.i.d. $\sim \mathrm{N}\left(\left(\begin{array}{c}0 \\ 0\end{array}\right),\left(\begin{array}{cc}\left(\sigma^{P}\right)^{2} & \rho \sigma^{P} \sigma^{S} \\ \rho \sigma^{P} \sigma^{S} & \left(\sigma^{S}\right)^{2}\end{array}\right)\right) i=0,1,2, r=1, \ldots, n_{i}$.

Define $T_{i}^{L}, i=1,2, L=P, S$, in (4) to be the t-statistic for testing the null hypothesis $H_{0 i}^{L}, i=$ $1,2, L=P, S$,

$$
T_{i}^{L}=\frac{\bar{Y}_{i \cdot}^{L}-\bar{Y}_{0 \cdot}^{L}-\delta^{L}}{\hat{\sigma}^{L} \sqrt{1 / n_{i}+1 / n_{0}}} \quad i=1,2, L=P, S
$$

where $\bar{Y}_{i}^{L}{ }^{L}=\frac{1}{n_{i}} \sum_{r=1}^{n_{i}} Y_{i r}^{L}, \hat{\sigma}^{L}=\sqrt{\frac{\sum_{i=0}^{2} \sum_{r=1}^{n_{i}}\left(Y_{i r}^{L}-\bar{Y}_{i .}^{L}\right)^{2}}{\sum_{i=0}^{2}\left(n_{i}-1\right)}}, i=0,1,2, L=P, S$.

The purpose of dose-response studies is to find which doses are effective. Control of multiple testing error rate should control the probability of incorrectly inferring a dose is efficacious for 
some endpoint when in fact it is not. In this situation, controlling the False Discovery Rate (FDR) will not control this probability (see [9]), while controlling the Familywise Error Rate (FWER) strongly for appropriately formulated null hypotheses will. We thus consider methods that strongly control FWER in this article.

With four null hypotheses in (1) and (2), a straight-forward application of the closed testing principle would test $2^{4}-1=15$ intersection null hypotheses to control FWER. However, we will show that if inferences are ordered by both endpoints and doses, then testing only four (disjoint) null hypotheses according to the partitioning principle controls FWER. These hypotheses are tested in three steps, with multiplicity adjustment involved in only one of the steps. When there are $k>2$ doses and $m>2$ endpoints, a similar step-down procedure could in theory be developed following the same principle.

\section{Multiple tests construction using the partitioning principle}

The purpose of this article is to demonstrate how to use the partitioning principle, a fundamental multiple tests constructing technique, to construct multiple tests when there are multiple doses and multiple endpoints. We illustrate the idea with the two hypotheses in (1), concerning the primary endpoint first.

\subsection{Closed testing to step-down doses according to sample responses}

The closed testing technique of [10] tests all possible non-empty intersections of the hypotheses in (1), leading to the three hypotheses in (5), each at level- $\alpha$ :

$$
H_{0}^{\cap}: \quad \theta_{1} \leq \delta \text { and } \theta_{2} \leq \delta \quad \text { (Neither dose is efficacious) }
$$




$$
\begin{aligned}
& H_{01}: \quad \theta_{1} \leq \delta \quad \text { (Low dose is not efficacious) } \\
& H_{02}: \quad \theta_{2} \leq \delta \quad \text { (High dose is not efficacious) }
\end{aligned}
$$

The logical implications of testing are:

- If $H_{0}^{\cap}$ is not rejected, no inference is given even if $H_{01}$ or $H_{02}$ or both are rejected, because $H_{0}^{\cap}$ implies $H_{01}$ and $H_{02}$.

- If only $H_{0}^{\cap}$ and $H_{01}\left(H_{02}\right)$ are rejected but not $H_{02}\left(H_{01}\right)$, then infer low (high) dose is efficacious.

- If all three hypotheses are rejected, then infer both doses are efficacious.

We use t-statistics $T_{1}$ and $T_{2}$ defined in (4) (with superscript $P$ dropped) to test the three intersection hypotheses in (5) with critical values $d_{\alpha, 2, \nu}$ (the upper $\alpha$ quantile of Dunnett distribution with 2 and $\nu=\sum_{i=0}^{2}\left(n_{i}-1\right)$ degrees of freedom) and $t_{\alpha, \nu}$ (the upper $\alpha$ quantile of $\mathrm{t}$ distribution with $\nu$ degrees of freedom). Let (1), (2) denote the random indices such that $T_{(1)}<T_{(2)}$, then the rejection rules can be shown in Table 2 .

Table 2: Decision rules for closed testing with two doses single primary endpoint

\begin{tabular}{|l|l|l|}
\hline Hypothesis & Rejection rule & Test level \\
\hline$H_{0}^{\cap}: \theta_{1} \leq \delta$ and $\theta_{2} \leq \delta$ & $T_{(2)}>d_{\alpha, 2, \nu}$ & $\alpha$ \\
\hline$H_{01}: \theta_{1} \leq \delta$ & $T_{1}>t_{\alpha, \nu}$ & $\alpha$ \\
\hline$H_{02}: \theta_{2} \leq \delta$ & $T_{2}>t_{\alpha, \nu}$ & $\alpha$ \\
\hline
\end{tabular}

Since the critical values satisfy $d_{\alpha, 2, \nu}>t_{\alpha, \nu}$, a step-down procedure with sample-determined steps exists, as follows: 
- Step 1: If $T_{(2)}>d_{\alpha, 2, \nu}$, infer dose (2) is efficacious and go to step 2; else stop.

- Step 2: If $T_{(1)}>t_{\alpha, \nu}$, infer dose (1) is efficacious and stop; else stop.

\subsection{Partition to step-down doses}

If higher dosage is expected to be more efficacious, then one may choose to always test the high dose first. If high dose is effective, then proceed to test low dose; otherwise, stop. We can formally state this pre-determined sequence of testing as:

Condition A (order in doses): The low dose can not be claimed efficacious unless the high dose has shown evidence of efficacy.

The partitioning principle of [11] and [12] is a general principle for constructing multiple tests. Under condition $A$, [13] partition the null space, $\left\{\boldsymbol{\theta} \in \mathbb{R}^{2} \mid \theta_{1} \leq \delta\right.$ or $\left.\theta_{2} \leq \delta\right\}$ into two disjoint subspaces $\left\{\boldsymbol{\theta} \in \mathbb{R}^{2} \mid \theta_{2} \leq \delta\right\}$ and $\left\{\boldsymbol{\theta} \in \mathbb{R}^{2} \mid \theta_{1} \leq \delta\right.$ and $\left.\theta_{2}>\delta\right\}$ corresponding to the two hypotheses:

$$
\begin{aligned}
& H_{02}^{\downarrow}: \quad \theta_{2} \leq \delta \quad \text { (High dose is not efficacious) } \\
& H_{01}^{\downarrow}: \quad \theta_{1} \leq \delta \text { and } \theta_{2}>\delta \quad(\text { Low dose is not efficacious but high dose is) }
\end{aligned}
$$

The logical implications of testing are:

- If $H_{02}^{\downarrow}$ is not rejected (regardless of $H_{01}^{\downarrow}$ ), then no inference is given, since "neither dose is efficacious" (which is contained in $H_{02}^{\downarrow}$ ) is not rejected.

- If $H_{02}^{\downarrow}$ and $H_{01}^{\downarrow}$ are rejected, then since the union of $H_{02}^{\downarrow}$ and $H_{01}^{\downarrow}$ is "either low dose or high dose is not efficacious", the implication is "both high dose and low dose are efficacious".

- If $H_{02}^{\downarrow}$ is rejected but $H_{01}^{\downarrow}$ is not rejected, then one infers high dose is efficacious. 
The interesting thing is, in testing $H_{0 i}^{\downarrow}, i=1,2$ simultaneously, no multiplicity adjustment is needed to control FWER, the probability of rejecting any true null hypothesis. This is because the null spaces of $H_{0 i}^{\downarrow}, i=1,2$ are disjoint. In other words, at most one null hypothesis can be true: it cannot be the case that high dose is ineffective $\left(H_{02}\right)$ and that high dose is effective but low dose is ineffective $\left(H_{01}\right)$, for example. We thus test each $H_{0 i}^{\downarrow}, i=1,2$, at level- $\alpha$.

Level $\alpha$ tests for each $H_{0 i}^{\downarrow}, i=1,2$, are of course not unique. Note, however, a level- $\alpha$ test for $H_{01}: \theta_{1} \leq \delta$ is also a level- $\alpha$ test for $H_{01}^{\downarrow}: \theta_{1} \leq \delta$ and $\theta_{2}>\delta$. For example, a test which rejects no more than $5 \%$ of the time when low dose is ineffective, regardless of whether high dose is effective, will reject no more than $5 \%$ of the time in particular when low dose is ineffective and high dose is effective.

So the simplest level- $\alpha$ test for $H_{0 i}^{\downarrow}$ is to use a one-sided two-sample size $\alpha$ t-test based on $T_{i}, i=1,2$ defined in (4) for each $H_{0 i}^{\downarrow}$ as shown in Table 3.

Table 3: Decision rules for partition testing to step-down doses

\begin{tabular}{|l|l|l|}
\hline Hypothesis & Rejection rule & Test level \\
\hline$H_{0}^{\cap}: \theta_{2} \leq \delta$ & $T_{2}>t_{\alpha, \nu}$ & $\alpha$ \\
\hline$H_{01}: \theta_{1} \leq \delta$ and $\theta_{2}>\delta$ & $T_{1}>t_{\alpha, \nu}$ & $\alpha$ \\
\hline
\end{tabular}

In terms of the rejection rules in Table 3, the pre-determined D-steps (D stands for doses) proceeds as follows.

- Step 1: If $T_{2}>t_{\alpha, \nu}$, infer high dose is efficacious and go to step 2; else stop.

- Step 2: If $T_{1}>t_{\alpha, \nu}$, infer low dose is efficacious and stop; else stop. 
Note that, even though the method above controls FWER regardless of whether the shape of the true response function, it is recommended only when the response is expected to be monotonically increasing (for otherwise it might stop too soon and miss an efficacious dose).

\section{Partition testing with two doses and two endpoints}

Suppose the dose-response study has both a primary endpoint and a secondary endpoint, so that the family of null hypotheses of interest includes both those in (1) and (2). We will show that, in addition to stepping through doses, the partitioning principle can also be used to derived multiple tests that step through endpoints in a pre-determined sequence.

\subsection{Partition to step-down endpoints}

Multiple testing procedures for dose-response studies with a primary endpoint and hierarchically ordered secondary endpoints were developed in [7]. In the case of two doses and two endpoints (one secondary endpoint), the procedure has to satisfy the condition that for the same dose, the secondary endpoint is tested only if primary endpoint is claimed to be efficacious, which is formally stated as:

Condition B (order in endpoints): For the same dose, a secondary endpoint can not be claimed efficacious unless its primary endpoint has been shown to be efficacious.

Within each endpoint, Dunnett's method was used in [7] to adjust multiplicity for multiple doses. The FWER of the whole procedure was controlled at $\alpha$ using the principle of closed testing. Figure 1 is a graphical representation of their procedure with two doses and two endpoints.

The procedure in [7] can be reproduced by partitioning the parameter space with constraints on 
Figure 1: Decision process in [7]

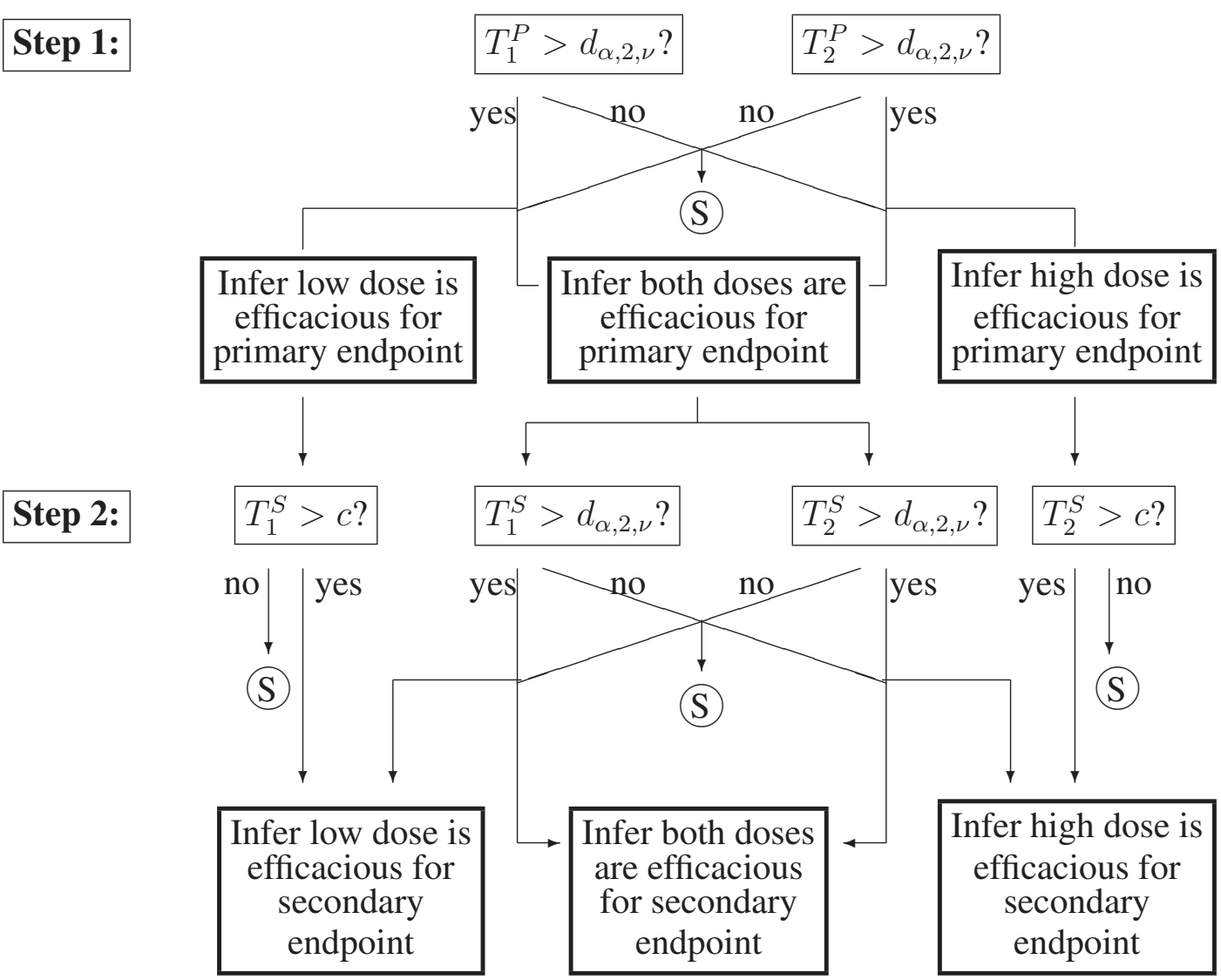

S means "Stop", $c$ is calculated based on sample correlation between endpoints.

ordered endpoints for each dose, similar to partitioning to step-down through the doses technique as illustrated in section 4. For example, for high dose, we would test the following two hypotheses in a step-down fashion (pre-determined E-steps).

$$
H_{02}^{P \downarrow}: \quad \theta_{2}^{P} \leq \delta^{P} \quad \text { (Primary endpoint is not effective) }
$$

$H_{02}^{S \downarrow}: \quad \theta_{2}^{S} \leq \delta^{S}$ and $\theta_{2}^{P}>\delta^{P} \quad$ (Secondary endpoint is not efficacious, but primary endpoint is) 


\subsection{Partitioning to step-down both doses and endpoints}

The procedure developed in [7] steps through the endpoints in a pre-determined sequence, satisfying condition $B$, but not necessarily steps through the doses in a pre-determined sequence.

To step through both doses and endpoints, we test the following four partition hypotheses in a step-down fashion (pre-determined DE-steps):

- Step 1: Test $H_{02}^{P \downarrow}: \theta_{2}^{P} \leq \delta^{P}$ (High dose is not effective for primary endpoint).

- If rejected, infer high dose for the primary endpoint is effective and proceed to step 2; else stop.

- Step 2: Test two hypotheses:

$H_{01}^{P \downarrow}: \theta_{1}^{P} \leq \delta^{P}$ and $\theta_{2}^{P}>\delta^{P}$ (Low dose is not effective, but high dose is effective for primary endpoint)

$H_{02}^{S \downarrow}: \theta_{2}^{S} \leq \delta^{S}$ and $\theta_{2}^{P}>\delta^{P}$ (High dose is not effective for secondary endpoint, but is effective for primary endpoint)

- If both hypotheses are rejected, infer both doses are effective for primary endpoint, high dose is also effective for secondary endpoint and proceed to the next step.

- Otherwise, if only $H_{01}^{P \downarrow}$ is rejected, infer both doses are effective for primary endpoint and stop.

- If only $H_{02}^{S \downarrow}$ is rejected, infer high dose is effective for both endpoints and stop.

- Step 3: Test $H_{01}^{S \downarrow}: \theta_{1}^{S} \leq \delta^{S}$ and $\theta_{2}^{S}>\delta^{S}$ and $\theta_{1}^{P}>\delta^{P}$ and $\theta_{2}^{P}>\delta^{P}$ (Low dose is not effective for secondary endpoint, but is effective for primary endpoint and high dose is 
effective for both endpoints.)

- If rejected, infer both doses are effective for both endpoints and stop. Otherwise stop.

The direction of these steps is presented in Table 4:

Table 4: Direction of the pre-determined DE-steps

\begin{tabular}{|c|ccc|}
\hline Endpoint $\backslash$ Dose & Low & & High \\
\hline Primary & Step 2 & $\leftarrow$ & Step 1 \\
& $\downarrow$ & & $\downarrow$ \\
Secondary & Step 3 & $\leftarrow$ Step 2 \\
\hline
\end{tabular}

${ }^{\mathrm{b}}$ Proceed only if both hypotheses in step 2 are rejected

\subsubsection{Decision tree}

The rejection rules for these hypotheses in each step are presented in Table 5 in the form of usual t-statistics $T_{i}^{L}, i=1,2, L=P, S$, as defined in (4).

Table 5: Critical values for the pre-determined DE-steps

\begin{tabular}{|l|l|l|l|}
\hline Step & Hypothesis & Rejection rule & Test level \\
\hline 1 & $H_{02}^{P \downarrow}: \theta_{2}^{P} \leq \delta^{P}$ & $T_{2}^{P}>c_{1}$ & $\alpha$ \\
\hline 2 & $H_{01}^{P \downarrow}: \theta_{1}^{P} \leq \delta^{P}$ and $\theta_{2}^{P}>\delta^{P}$ & $T_{1}^{P}>c_{2}$ & $\mathrm{MA}^{\mathrm{c}}$ \\
& $H_{02}^{S \downarrow}: \theta_{2}^{S} \leq \delta^{S}$ and $\theta_{2}^{P}>\delta^{P}$ & $T_{2}^{S}>c_{2}$ & $\mathrm{MA}$ \\
\hline 3 & $H_{01}^{S \downarrow}: \theta_{1}^{S} \leq \delta^{S}$ and $\theta_{2}^{S}>\delta^{S}$ and $\theta_{1}^{P}>\delta^{P}$ and $\theta_{2}^{P}>\delta^{P}$ & $T_{1}^{S}>c_{1}$ & $\alpha$ \\
\hline
\end{tabular}

${ }^{\mathrm{c}}$ Multiplicity adjustment needed 
For step 1 and 3, the simplest choice of critical value $c_{1}$ is $t_{\alpha, \nu}$. For step 2 , since the null spaces of $H_{01}^{P \downarrow}$ and $H_{02}^{S \downarrow}$ are not disjoint, we need to adjust for multiplicity to make the FWER for the whole procedure controlled at level $\alpha$.

The decision tree of this step-down procedure with pre-determined DE-steps is presented in Figure 2.

Figure 2: Decision tree for pre-determined DE-steps

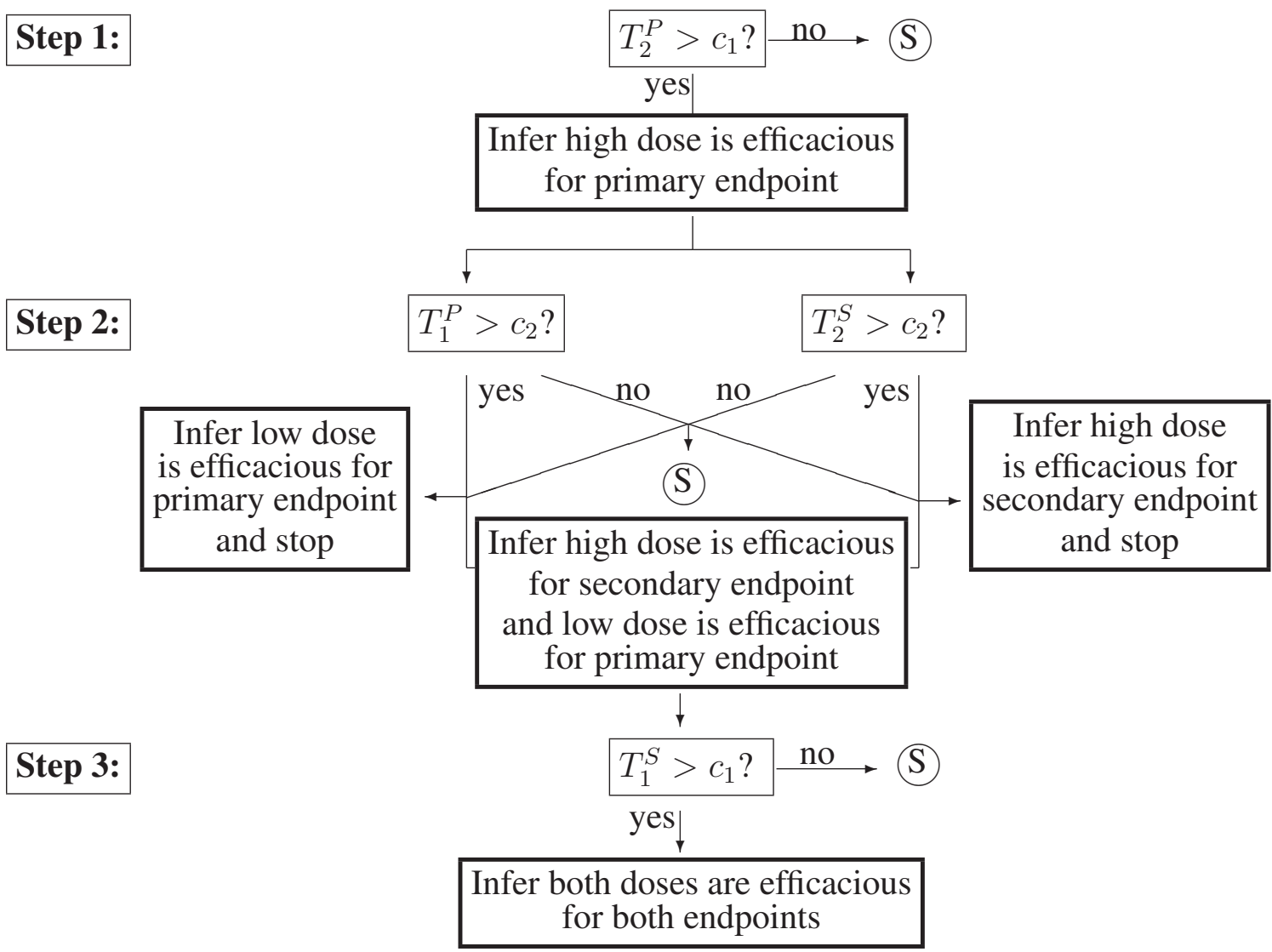

\subsubsection{Computing the critical value $c_{2}$}

According to Table 5, we need to find the critical value $c_{2}$ accounted for multiplicity for the two hypotheses in step 2. That is, one needs to focus on the joint distribution of $\left(T_{1}^{P}, T_{2}^{S}\right)$. Notice 
that between doses, the observations are independent, but within a dose, observations between endpoints are dependent, the numerators as well as the denominators of $T_{1}^{P}$ and $T_{2}^{S}$ are correlated through endpoints for the same dose. For one-sided tests, with the rejection rule of the form in (7), the supremum of the probability is obtained at the boundary values (involving $\theta_{1}^{P}$ and $\theta_{2}^{S}$ only, since neither $T_{1}^{P}$ nor $T_{2}^{S}$ involves $\theta_{2}^{P}$ ). The critical value $c_{2}$ needs to satisfy:

$$
\begin{aligned}
1-\alpha & \leq 1-\sup _{\theta_{1}^{P} \leq \delta^{P} \text { and } \theta_{2}^{S} \leq \delta^{S}} \operatorname{Pr}_{\rho}\left\{T_{1}^{P}>c_{2} \text { or } T_{2}^{S}>c_{2}\right\} \\
& =\operatorname{Pr}_{\rho}\left\{\frac{\bar{Y}_{1 \cdot}^{P}-\bar{Y}_{0 \cdot}^{P}-\theta_{1}^{P}}{\hat{\sigma}^{P} \sqrt{1 / n_{1}+1 / n_{0}}} \leq c_{2} \text { and } \frac{\bar{Y}_{2 \cdot}^{S}-\bar{Y}_{0 \cdot}^{S}-\theta_{2}^{S}}{\hat{\sigma}^{S} \sqrt{1 / n_{2}+1 / n_{0}}} \leq c_{2}\right\} \\
& =\operatorname{Pr}_{\rho}\left\{\frac{Z_{1}}{\hat{\sigma}^{P} / \sigma^{P}} \leq c_{2} \text { and } \frac{Z_{2}}{\hat{\sigma}^{S} / \sigma^{S}} \leq c_{2}\right\} \\
& =\int_{0}^{\infty} \int_{0}^{\infty} \operatorname{Pr}_{\rho}\left\{Z_{1} \leq c_{2} s_{1} \text { and } Z_{2} \leq c_{2} s_{2}\right\} \gamma_{\rho, \nu}\left(s_{1}, s_{2}\right) d s_{1} d s_{2}
\end{aligned}
$$

where $\left(Z_{1}, Z_{2}\right)=\left(\frac{\bar{Y}_{1 \cdot}^{P}-\bar{Y}_{0 \cdot}^{P}-\theta_{1}^{P}}{\sigma^{P} \sqrt{1 / n_{1}+1 / n_{0}}}, \frac{\bar{Y}_{2 \cdot}^{S}-\bar{Y}_{0 .}^{S}-\theta_{2}^{S}}{\sigma^{S} \sqrt{1 / n_{2}+1 / n_{0}}}\right)$ with mean $\mathbf{0}=(0,0)$, variance 1 and covariance $\rho /\left(\sqrt{\left(n_{0} / n_{1}+1\right)\left(n_{0} / n_{2}+1\right)}\right)(=\rho / 2$ in the balanced case $), \gamma_{\rho, \nu}\left(s_{1}, s_{2}\right)$ is the joint density of $\left(\frac{\hat{\sigma}^{P}}{\sigma^{P}}, \frac{\hat{\sigma}^{S}}{\sigma^{S}}\right)$.

Note that the definition of a bivariate $t$ distribution, as originally introduced by [14] and computed by the ProbMC function in SAS and the qmvnorm function in the R package mvtnorm, requires the denominators in (7) to be the same random variable $\hat{\sigma}^{P} \equiv \hat{\sigma}^{S}$, which is not the case here. In fact, computing (7) as if the distribution were bivariate t overestimates the probability, resulting in somewhat liberal critical values, as we will demonstrate. To avoid confusion, we call the distribution of $\left(T_{1}^{P}, T_{2}^{S}\right)$ the dual $t$ distribution. (This distribution was studied by [15].)

The probability (8) involves the unknown parameter $\rho$. Below we describe five ways of approximating (8). The first four methods (normal, bivariate $t$, dual $t$ and independent standard errors) require $\rho$ be known or approximated. The last method computes by pretending $T_{1}^{P}$ and $T_{2}^{S}$ are 
independent, which we prove results in a conservative critical value.

\section{Normal approximation}

If the sample sizes $n_{1}, n_{2}$ are large, by Slutsky's Theorem, $\hat{\sigma}^{P} / \sigma^{P}$ and $\hat{\sigma}^{S} / \sigma^{S}$ converge in probability to one, then the joint distribution of $\left(T_{1}^{P}, T_{2}^{S}\right)$ (under the true mean difference) is asymptotically bivariate normal as $\left(Z_{1}, Z_{2}\right)$.

\section{Bivariate t distribution}

Assume $\frac{\hat{\sigma}^{P}}{\sigma^{P}}$ and $\frac{\hat{\sigma}^{S}}{\sigma^{S}}$ are perfectly correlated, or they are the same, the situation coincides with the bivariate $t$ case. Specifically, the probability in (7) reduces to

$$
\int_{0}^{\infty} \operatorname{Pr}_{\rho}\left\{Z_{1} \leq c_{2} s \text { and } Z_{2} \leq c_{2} s\right\} \gamma_{\nu}(s) d s
$$

where $\gamma_{\nu}(s)$ is the density of $\sqrt{\chi_{\nu}^{2} / \nu}$, and $\nu=\sum_{i=0}^{2}\left(n_{i}-1\right)$ is the degrees of freedom.

\section{Dual t distribution}

It can be shown that $\frac{\hat{\sigma}^{P}}{\sigma^{P}}$ and $\frac{\hat{\sigma}^{S}}{\sigma^{S}}$ are diagonal elements of a Wishart random matrix (see Appendix). We use simulations from a Wishart distribution (which depends on $\rho$, the correlation between endpoints from a single individual) and Monte Carlo methods to approximate the probability based on exact dual $\mathrm{t}$ distribution of the test statistics $\left(T_{1}^{P}, T_{2}^{S}\right)$.

\section{Independent Standard Errors $\hat{\sigma}^{P}, \hat{\sigma}^{S}$}

The difficulty in computing (7) is caused, in part, by pooling data across doses in estimating the standard errors $\sigma^{P}, \sigma^{S}$, resulting in the denominators of test statistics $\left(T_{1}^{P}, T_{2}^{S}\right),\left(\frac{\hat{\sigma}^{P}}{\sigma^{P}}, \frac{\hat{\sigma}^{S}}{\sigma^{S}}\right)$ being 
correlated. One can obtain a conservative upper bound of $c_{2}$ (see Theorem 1 ) in solving $(9)=1-\alpha$ for $c_{2}$ by pretending $\frac{\hat{\sigma}^{P}}{\sigma^{P}}$ and $\frac{\hat{\sigma}^{S}}{\sigma^{S}}$ are independent, where (9) can be viewed as a simplified version of (8).

$$
\int_{0}^{\infty} \int_{0}^{\infty} \operatorname{Pr}_{\rho}\left\{Z_{1} \leq c_{2} s_{1} \text { and } Z_{2} \leq c_{2} s_{2}\right\} \gamma_{\nu}\left(s_{1}\right) \gamma_{\nu}\left(s_{2}\right) d s_{1} d s_{2}
$$

Theorem 1 Probability (7) is greater when $\frac{\hat{\sigma}^{P}}{\sigma^{P}}$ and $\frac{\hat{\sigma}^{S}}{\sigma^{S}}$ are correlated than when they are independent.

Proof of Theorem 1: We first condition on $Z_{1}$ and $Z_{2}$, and note $c_{2}>0$

$$
\begin{aligned}
& \operatorname{Pr}_{\rho}\left\{\frac{Z_{1}}{\hat{\sigma}^{P} / \sigma^{P}} \leq c_{2} \text { and } \frac{Z_{2}}{\hat{\sigma}^{S} / \sigma^{S}} \leq c_{2}\right\} \\
= & \mathrm{E}_{\left(Z_{1}, Z_{2}\right)}\left[\operatorname{Pr}_{\rho}\left\{\frac{z_{1}}{\hat{\sigma}^{P} / \sigma^{P}} \leq c_{2} \text { and } \frac{z_{2}}{\hat{\sigma}^{S} / \sigma^{S}} \leq c_{2} \mid\left(Z_{1}, Z_{2}\right)=\left(z_{1}, z_{2}\right)\right\}\right] \\
= & \mathrm{E}_{\left(Z_{1}, Z_{2}\right)}\left[\operatorname{Pr}_{\rho}\left\{\frac{z_{1}}{\hat{\sigma}^{P} / \sigma^{P}} \leq c_{2} \text { and } \frac{z_{2}}{\hat{\sigma}^{S} / \sigma^{S}} \leq c_{2}\right\}\right] \\
= & \mathrm{E}_{\left(Z_{1}, Z_{2}\right)}\left[\operatorname{Pr}_{\rho}\left\{\hat{\sigma}^{P} / \sigma^{P} \geq \frac{z_{1}}{c_{2}} \text { and } \hat{\sigma}^{S} / \sigma^{S} \geq \frac{z_{2}}{c_{2}}\right\}\right]
\end{aligned}
$$

Since $\frac{\hat{\sigma}^{P}}{\sigma^{P}}$ and $\frac{\hat{\sigma}^{S}}{\sigma^{S}}$ are associated (see Theorem 6.1, [16]), for any given $Z_{1}$ and $Z_{2}$

$$
\operatorname{Pr}_{\rho}\left\{\hat{\sigma}^{P} / \sigma^{P} \geq \frac{z_{1}}{c_{2}} \text { and } \hat{\sigma}^{S} / \sigma^{S} \geq \frac{z_{2}}{c_{2}}\right\} \geq \operatorname{Pr}\left\{\hat{\sigma}^{P} / \sigma^{P} \geq \frac{z_{1}}{c_{2}}\right\} \operatorname{Pr}\left\{\hat{\sigma}^{S} / \sigma^{S} \geq \frac{z_{2}}{c_{2}}\right\}
$$

The result follows.

Independent t statistics $T_{1}^{P}, T_{2}^{S}$

To further break down the four dimensional integral in (9), we add the assumption of independent numerators $\left(Z_{1}, Z_{2}\right)$ of $\left(T_{1}^{P}, T_{2}^{S}\right)$ in addition to independent denominators, which is reasonable since the correlation between $Z_{1}$ and $Z_{2}$ is only $\rho / 2$ in the balanced case. This method is more conservative then the previous method as shown in Theorem 2 . 
Theorem 2 The probability (9) assuming $\frac{\hat{\sigma}^{P}}{\sigma^{P}}$ and $\frac{\hat{\sigma}^{S}}{\sigma^{S}}$ are independent is greater than the probability assuming test statistics $\left(T_{1}^{P}, T_{2}^{S}\right)$ are independent. (This is equivalent to further assuming the numerators of $\left(T_{1}^{P}, T_{2}^{S}\right),\left(Z_{1}, Z_{2}\right)$ are independent. $)$

\section{Proof of Theorem 2:}

Assume independence of $\frac{\hat{\sigma}^{P}}{\sigma^{P}}$ and $\frac{\hat{\sigma}^{S}}{\sigma^{S}}$, we have

$$
\begin{aligned}
& \operatorname{Pr}_{\rho}\left\{\frac{Z_{1}}{\hat{\sigma}^{P} / \sigma^{P}} \leq c_{2} \text { and } \frac{Z_{2}}{\hat{\sigma}^{S} / \sigma^{S}} \leq c_{2}\right\} \\
= & \int_{0}^{\infty} \int_{0}^{\infty} \operatorname{Pr}_{\rho}\left\{Z_{1} \leq c_{2} s_{1} \text { and } Z_{2} \leq c_{2} s_{2}\right\} \gamma_{\nu}\left(s_{1}\right) \gamma_{\nu}\left(s_{2}\right) d s_{1} d s_{2} \\
& \text { since } \operatorname{Cov}\left(Z_{1}, Z_{2}\right)=\rho / \sqrt{\left(n_{0} / n_{1}+1\right)\left(n_{0} / n_{2}+1\right)}>0 \\
& \text { By Slepian's inequality (Corollary A.3.1 on p. 229 of [17]) } \\
\geq & \int_{0}^{\infty} \int_{0}^{\infty} \operatorname{Pr}\left\{Z_{1} \leq c_{2} s_{1}\right\} \operatorname{Pr}\left\{Z_{2} \leq c_{2} s_{2}\right\} \gamma_{\nu}\left(s_{1}\right) \gamma_{\nu}\left(s_{2}\right) d s_{1} d s_{2} \\
= & \int_{0}^{\infty} \operatorname{Pr}\left\{Z_{1} \leq c_{2} s_{1}\right\} \gamma_{\nu}\left(s_{1}\right) d s_{1} \int_{0}^{\infty} \operatorname{Pr}\left\{Z_{2} \leq c_{2} s_{2}\right\} \gamma_{\nu}\left(s_{2}\right) d s_{2} \\
= & \operatorname{Pr}\left\{\frac{Z_{1}}{\left.\hat{\sigma}^{P} / \sigma^{P} \leq c_{2}\right\} \operatorname{Pr}\left\{\frac{Z_{2}}{\hat{\sigma}^{S} / \sigma^{S}} \leq c_{2}\right\}}\right. \\
= & \operatorname{Pr}\left\{T_{1}^{P} \leq c_{2}\right\} \operatorname{Pr}\left\{T_{2}^{S} \leq c_{2}\right\} \square
\end{aligned}
$$

The degrees of conservatism for the methods introduced above are compared in terms of critical value $c_{2}$ for different combinations of correlation between endpoints $\rho$ and error degrees of freedom $\nu$ in Table 6. Critical values for the dual $\mathrm{t}$ and independent standard errors methods are based on 100,000 simulations.

As shown in Table 6, for each combination of $\rho$ and $\nu$, the normal approximation is somewhat liberal and the bivariate t approximation is slightly liberal. Assuming independence of $\frac{\hat{\sigma}^{P}}{\sigma^{P}}$ and $\frac{\hat{\sigma}^{S}}{\sigma^{S}}$ is slightly conservative, while assuming independence of $\mathrm{t}$ statistics is somewhat conservative. It should be noted that, in the setting of simultaneous efficacy and safety studies, [19] considered 
Table 6: Comparing conservatism in terms of critical value $c_{2}$

\begin{tabular}{|c|c|c|c|c|c|c|}
\hline \multirow[b]{2}{*}{$\nu$} & \multirow[b]{2}{*}{ Method } & \multicolumn{5}{|c|}{$\rho^{\mathrm{d}}$} \\
\hline & & 0 & 0.2 & 0.4 & 0.6 & 0.8 \\
\hline \multirow{4}{*}{50} & Bivariate $\mathrm{t}^{\mathrm{e}}$ & 2.0015 & 1.9972 & 1.9913 & 1.9833 & 1.9730 \\
\hline & Dual $\mathrm{t}^{\mathrm{f}}$ & 2.0026 & 1.9988 & 1.9934 & 1.9851 & 1.9741 \\
\hline & Ind. SE & 2.0028 & 1.9988 & 1.9940 & 1.9866 & 1.9771 \\
\hline & Ind. $\mathrm{T}$ & 2.0028 & 2.0028 & 2.0028 & 2.0028 & 2.0028 \\
\hline \multirow{4}{*}{100} & Bivariate $\mathrm{t}$ & 1.9777 & 1.9737 & 1.9682 & 1.9607 & 1.9507 \\
\hline & Dual t & 1.9783 & 1.9744 & 1.9692 & 1.9617 & 1.9513 \\
\hline & Ind. SE & 1.9783 & 1.9745 & 1.9694 & 1.9623 & 1.9527 \\
\hline & Ind. $\mathrm{T}$ & 1.9783 & 1.9783 & 1.9783 & 1.9783 & 1.9783 \\
\hline \multirow{4}{*}{200} & Bivariate $\mathrm{t}$ & 1.9660 & 1.9622 & 1.9569 & 1.9495 & 1.9398 \\
\hline & Dual t & 1.9659 & 1.9627 & 1.9572 & 1.9498 & 1.9401 \\
\hline & Ind. SE & 1.9664 & 1.9628 & 1.9573 & 1.9503 & 1.9408 \\
\hline & Ind. $\mathrm{T}$ & 1.9664 & 1.9664 & 1.9664 & 1.9664 & 1.9664 \\
\hline$\infty$ & Normal $^{\mathrm{g}}$ & 1.9545 & 1.9508 & 1.9456 & 1.9385 & 1.9289 \\
\hline
\end{tabular}

${ }^{\mathrm{d}}$ Note $\rho$ refers to the correlation between endpoints for a single individual. The correlation between numerators $\left(Z_{1}, Z_{2}\right)$ of the test statistics, is assumed to be $\rho / 2$ in the balanced case.

${ }^{\mathrm{e}}$ The critical values of bivariate $\mathrm{t}$ are obtained by qmvt function in R package mvtnorm.

${ }^{\mathrm{f}}$ Wishart is generated first using Bartlett's decomposition and then appropriately transformed (see [18]).

${ }^{\mathrm{g}}$ The bivariate normal quantiles are obtained by qmvnorm of R package mvtnorm. 
using the Bonferroni inequality and the bootstrap technique to compute such probabilities.

\section{Analysis of the anti-psychotic drug data}

We illustrate the step-down procedure with pre-determined DE-steps using the anti-psychotic drug example introduced at the beginning of the paper with $\delta^{P}=\delta^{S}=0$. Using pooled standard error from these three groups, the t-statistics corresponding to each dose and endpoint and the error degrees of freedom are:

$T_{1}^{P}=-1.7499, \quad T_{2}^{P}=-3.7318, \quad T_{1}^{S}=-0.2116, \quad T_{2}^{S}=-0.4212 \quad$ and $\quad \nu=151$.

If we want to control the FWER at 5\% level, then $c_{1}=t_{.05,151}=1.6550$, while $c_{2}=1.9702$, based on the independent $\mathrm{t}$ method (which is appropriate because the correlation is unknown). Note in this particular example, a lower score indicates better drug effect. To make it consistent with our hypotheses set up, we take the negatives of the t-statistics and then apply our procedure.

- Step 1: Is $3.7318>1.6550$ ? Yes, go to step 2.

- Step 2: Is $1.7499>1.9702$ or $0.4212>1.9702$ ? No, stop.

So infer high dose (600 mg/day) is efficacious for primary endpoint only, and no evidence of efficacy can be made for low dose ( $75 \mathrm{mg} /$ day $)$.

\section{Conclusion}

In this paper, we present a systematic way of constructing null hypotheses by partitioning the parameter space according to conditions that order inferences by dose (high to low), or endpoint (primary to secondary), or both. The hypotheses so constructed automatically form step-down 
procedures (pre-determined D-steps, E-steps, or DE-steps) and control the proper error rate at a pre-specified level. This way of tests construction can be generalized to situations with $k>2$ doses and $m>2$ endpoints. The technique is as follows. Start by writing down the null hypotheses whose rejections correspond to desired inferences on primary and secondary endpoints. Then, following stated conditions for the decision process, make each null hypothesis in a subsequent step disjoint from null hypotheses in previous steps (by removing from it the union of the null hypotheses whose rejections lead up to it). Adjust for multiplicity within a step (only) if the null hypotheses are not disjoint, but do not adjust for multiplicity between steps.

An important issue in multiple endpoints problems is how to deal with correlations among the test statistics induced by correlations among measurements on the multiple endpoints. We made a systematic study of liberalism and conservatism of five approximation methods.

Graphical representation makes the decision process clear and easy to understand. Multiplicity adjustment may be needed for some steps but not all. There are two general rules. First, if there are two or more statistics involved in one box, then multiplicity adjustment may be needed. Second, if there are two or more branches from a box, and the hypotheses corresponding to these boxes are not disjoint, then further multiplicity adjustment may be needed among those boxes.

\section{Acknowledgement}

Jason Hsu's research is supported by Grant No. DMS-0505519 from the U.S. National Science Foundation. Haiyan $\mathrm{Xu}$ motivated us to work on this problem, and we have had rather useful discussions with her, Frank Bretz, and John Lawrence. We also thank the reviewers for improving the paper with very helpful comments. 


\section{References}

1. ICH E10. Choice of Control Groups in Clinical Trials. CPMP (Committee for Propritary Medical Products), EMEA (The European Agency for the Evaluation of Medical Products), London, Draft ICH (International Conference on Harmonisation) Guideline ed., 1999. http://www.ifpma.org/pdfifpma/e10.pdf.

2. Temple RJ. Effect size - Can the effect be too small. Presentation at FDA Center for Drug Evaluation and Research, Cardiovascular and Renal Advisory Committee meeting, 2006. http://www.fda.gov/ohrms/dockets/ac/06/slides/2006-4215S1-02-FDA.ppt.

3. Ruberg SJ. Contrasts for identifying the minimum effective dose. Journal of the American Statistical Association 1989; 84(407): 816-822.

4. Ruberg SJ. Dose response studies.I. Some design considerations. Journal of Biopharmaceutical Statistics 1995; 5(1): 1-14.

5. Ruberg SJ. Dose response studies.II. Analysis and interpretation. Jouranl of Biopharmaceutical Statistics 1995; 5(1): 15-42.

6. Ruberg S, Cairns V. Providing evidence of efficacy for a new drug. Statistics in Medicine 1998; 17(15-16): 1813-1823.

7. Dmitrienko A, Offen W, Wang O, Xiao D. Gatekeeping procedures in dose-response clinical trials based on the Dunnett test. Pharmaceutical Statististics 2006; 5: 19-28.

8. Arvanitis L, Miller B, the Seroquel Trial 13 Study Group. Multiple fixed doses of "Sero- 
quel" (Quetiapine) in patients with acute exacerbation of Schizophrenia: A comparison with Haloperidol and placebo. Biological Psychiatry 1997; 42: 233-246.

9. Huang Y, Hsu JC. Hochberg's Step-up Method: Cutting Corners off Holm's Step-down Method. To appear in Biometrika 2007; 94.

10. Marcus R, Peritz E, Gabriel KR. On closed testing procedures with special reference to ordered analysis of variance. Biometrika 1976; 63: 655-660.

11. Stefansson G, Kim WC, Hsu JC. On confidence sets in multiple comparisons. In SS Gupta, JO Berger, editors, Statistical Decision Theory and Related Topics IV. Springer-Verlag, New York, 1988; 2: 89-104.

12. Finner H, Strassburger K. The partitioning principle: a powerful tool in multiple decision theory. Annals of Statistics 2002; 30: $1194-1213$.

13. Hsu JC, Berger RL. Stepwise confidence intervals without multiplicity adjustment for doseresponse and toxicity studies. Journal of the American Statistical Association 1999; 94(446): $468-482$.

14. Dunnett CW, Sobel M. A bivariate generalization of Student's $t$-distribution, with tables for certain special cases. Biometrika 1954; 41: 153-169.

15. Siddiqui MM. A bivariate t distribution. The Annals of Mathematical Statistics 1967; 38(1): $162-166$.

16. Karlin S, Rinott Y. Total positivity properties of absolute value multinormal variables with 
applications to confidence interval estimates and related probabilistic inequalities. The Annals of Statistics 1981; 9(5): 1035-1049.

17. Hsu JC. Multiple Comparisons:Theory and Methods. Chapman and Hall, 1996.

18. Johnson ME. Multivariate Statistical Simulation. Wiley, 1987.

19. Tamhane AC, Logan BR. Multiple test procedures for identifying the minimum effective and maximum safe doses of a drug. Journal of the American Statistical Association 2002; 97(457): 293-301.

20. Johnson RA, Wichern DW. Applied Multivariate Statistical Analysis. Prentice Hall, 5 ed., 2002. 


\section{Appendix}

\section{Model for dose-response studies with two endpoints}

Suppose we have samples $Y_{0 r}^{\prime}=\left(Y_{0 r}^{P}, Y_{0 r}^{S}\right), r=1, \ldots, n_{0}$ from a placebo group, and $Y_{1 r}^{\prime}=$ $\left(Y_{1 r}^{P}, Y_{1 r}^{S}\right), r=1, \ldots, n_{1}, Y_{2 r}^{\prime}=\left(Y_{2 r}^{P}, Y_{2 r}^{S}\right), r=1, \ldots, n_{2}$ from two dose groups, measuring two endpoints. Let $\boldsymbol{\mu}_{0}=\left(\mu_{0}^{P}, \mu_{0}^{S}\right)^{\prime}, \boldsymbol{\mu}_{1}=\left(\mu_{1}^{P}, \mu_{1}^{S}\right)^{\prime}, \boldsymbol{\mu}_{2}=\left(\mu_{2}^{P}, \mu_{2}^{S}\right)^{\prime}$ be the group means, $\Sigma=$ $\left(\begin{array}{cc}\left(\sigma^{P}\right)^{2} & \rho \sigma^{P} \sigma^{S} \\ \rho \sigma^{P} \sigma^{S} & \left(\sigma^{S}\right)^{2}\end{array}\right)$ be the covariance matrix of $\epsilon_{n}$, the $n$th row of $\boldsymbol{\epsilon}$, and $\mathbf{1}_{n}$ be a column vector of $1 \mathrm{~s}$ of length $n$. The model can be written in the following form:

$$
\mathbf{Y}=\left(\begin{array}{c}
\mathbf{Y}_{\mathbf{0}} \\
\mathbf{Y}_{\mathbf{1}} \\
\mathbf{Y}_{\mathbf{2}}
\end{array}\right)=\left(\begin{array}{cc}
Y_{01}^{P} & Y_{01}^{S} \\
\vdots & \vdots \\
Y_{0 n_{0}}^{P} & Y_{0 n_{0}}^{S} \\
Y_{11}^{P} & Y_{11}^{S} \\
\vdots & \vdots \\
Y_{1 n_{1}}^{P} & Y_{1 n_{1}}^{S} \\
Y_{21}^{P} & Y_{21}^{S} \\
\vdots & \vdots \\
Y_{2 n_{2}}^{P} & Y_{2 n_{2}}^{S}
\end{array}\right)=\left(\begin{array}{ccc}
1 & 0 & 0 \\
\vdots & \vdots & \vdots \\
1 & 0 & 0 \\
0 & 1 & 0 \\
\vdots & \vdots & \vdots \\
0 & 1 & 0 \\
0 & 0 & 1 \\
\vdots & \vdots & \vdots \\
0 & 0 & 1
\end{array}\right)\left(\begin{array}{cc}
\mu_{0}^{P} & \mu_{0}^{S} \\
\mu_{1}^{P} & \mu_{1}^{S} \\
\mu_{2}^{P} & \mu_{2}^{S}
\end{array}\right)+\boldsymbol{\epsilon}=\mathbf{X}\left(\begin{array}{c}
\mu_{0}^{\prime} \\
\mu_{1}^{\prime} \\
\mu_{2}^{\prime}
\end{array}\right)+\boldsymbol{\epsilon}
$$

The projection matrix $\mathbf{H}=\left(\begin{array}{ccc}\frac{1}{n_{0}} \mathbf{1}_{n_{0}} \mathbf{1}_{n_{0}}^{\prime} & 0 & 0 \\ 0 & \frac{1}{n_{1}} \mathbf{1}_{n_{1}} \mathbf{1}_{n_{1}}^{\prime} & 0 \\ 0 & 0 & \frac{1}{n_{2}} \mathbf{1}_{n_{2}} \mathbf{1}_{n_{2}}^{\prime}\end{array}\right)=\left(\begin{array}{ccc}H_{0} & 0 & 0 \\ 0 & H_{1} & 0 \\ 0 & 0 & H_{2}\end{array}\right)$. 
$\mathbf{I}-\mathbf{H}=\left(\begin{array}{ccc}I-H_{0} & 0 & 0 \\ 0 & I-H_{1} & 0 \\ 0 & 0 & I-H_{2}\end{array}\right)$ and $N \hat{\Sigma}=N \mathbf{Y}^{\prime}(\mathbf{I}-\mathbf{H}) \mathbf{Y}=N \sum_{i=0}^{2} \mathbf{Y}_{\mathbf{i}}^{\prime}\left(I-H_{i}\right) \mathbf{Y}_{\mathbf{i}}$

By Result 7.10. on p. 390 of [20], $N \hat{\Sigma}$ follows the Wishart distribution $W_{2}(N-3, \Sigma)$, where $N=\sum_{i=0}^{2} n_{i}$, and $N-3$ is the degrees of freedom.

Notice, the diagonal elements of $N \hat{\Sigma}$ are $(N-3)\left(\hat{\sigma}^{P}\right)^{2}$ and $(N-3)\left(\hat{\sigma}^{S}\right)^{2}$, where $\hat{\sigma}^{P}=$ $\sqrt{\frac{\sum_{i=0}^{2} \sum_{r=1}^{n_{i}}\left(Y_{i r}^{P}-\bar{Y}_{i .}^{P}\right)^{2}}{N-3}}$ and $\hat{\sigma}^{S}=\sqrt{\frac{\sum_{i=0}^{2} \sum_{r=1}^{n_{i}}\left(Y_{i r}^{S}-\bar{Y}_{i}^{S}\right)^{2}}{N-3}}$. To obtain the joint distribution of $\left(\frac{\hat{\sigma}^{P}}{\sigma^{P}}, \frac{\hat{\sigma}^{S}}{\sigma^{S}}\right)$, let $U=\operatorname{diag}\left\{\left(\sigma^{P}\right)^{-1},\left(\sigma^{S}\right)^{-1}\right\}$, then $N U^{\prime} \hat{\Sigma} U \sim W_{2}\left(N-3,\left(\begin{array}{cc}1 & \rho \\ \rho & 1\end{array}\right)\right)$ with diagonal elements $(N-3)\left(\frac{\hat{\sigma}^{P}}{\sigma^{P}}\right)^{2}$ and $(N-3)\left(\frac{\hat{\sigma}^{S}}{\sigma^{S}}\right)^{2}$. 